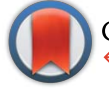

CrossMark

Cite this: Soft Matter, 2014, 10, 8376

DOI: $10.1039 / c 4 s m 90126 f$

www.rsc.org/softmatter

\section{Correction: Structure-delivery relationships of lysine-based gemini surfactants and their lipoplexes}

Mark Damen, ${ }^{a}$ Edgar Cristóbal-Lecina, ${ }^{a}$ Glòria Colom Sanmartí, ${ }^{a}$ Stijn F. M. van Dongen, ${ }^{a}$ Cristina L. García Rodríguez, ${ }^{a}$ Igor P. Dolbnya,,$^{\mathrm{b}}$ Roeland J. M. Nolte ${ }^{a}$ and Martin C. Feiters*a

Correction for 'Structure-delivery relationships of lysine-based gemini surfactants and their lipoplexes' by Mark Damen et al., Soft Matter, 2014, 10, 5702-5714.

There were errors in Table 2. The corrected version of Table 2 appears below:

Table 2 SAXS results for liposomes and lipoplexes of $\mathrm{R}^{1}(\mathrm{CO})$-Lys-H-n $\left(d=2 \pi / q_{001}\right.$, lamellar spacing; $a=4 \pi / \sqrt{ } 3 q_{100}$, hexagonal or columnar spacing) at $25^{\circ} \mathrm{C}$. OI, C18:1(Z) tails; El, C18:1(E) tails; St, saturated C18 tails

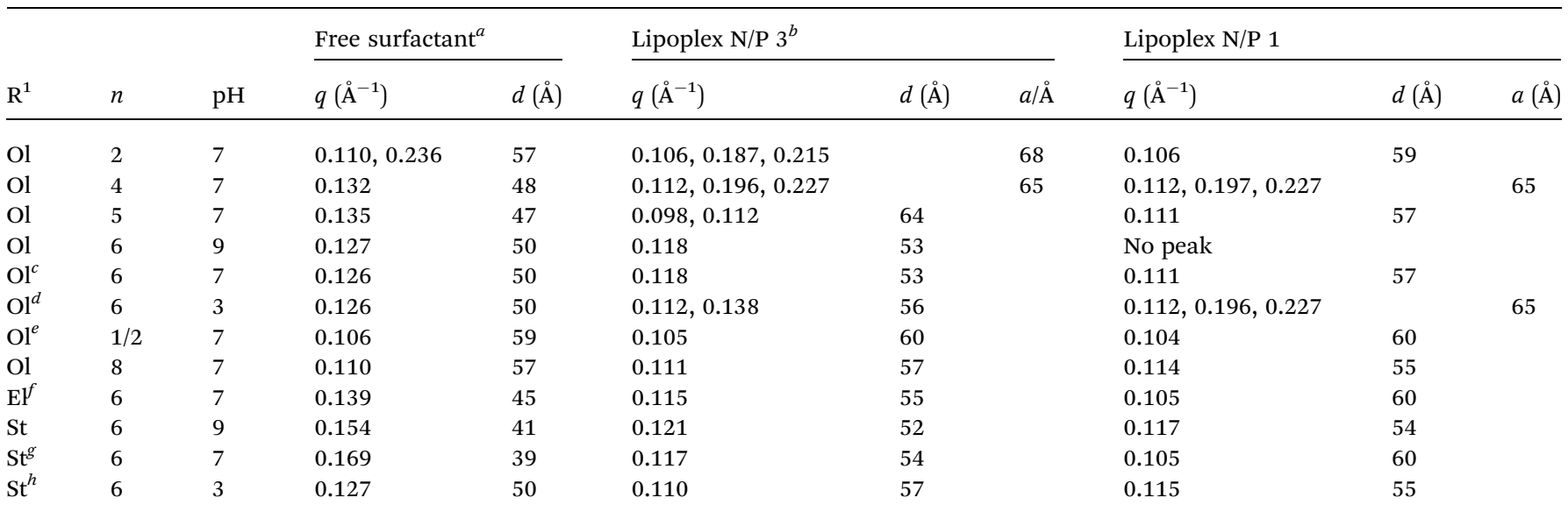

${ }^{a}$ Fig. 9a. ${ }^{b}$ Fig. 9b. ${ }^{c}$ Fig. 10a. ${ }^{d}$ Fig. 10b. ${ }^{e} n=1 / 2$ 'half-gemini' Ol-Lys-NHEt. ${ }^{f}$ Fig. $10{ }^{g}{ }^{g}$ Fig. 10 d. ${ }^{h}$ Fig. 10 e.

The Royal Society of Chemistry apologises for these errors and any consequent inconvenience to authors and readers.

\footnotetext{
${ }^{a}$ Radboud University Nijmegen, Institute for Molecules and Materials, Heijendaalseweg 135, 6525 AJ Nijmegen, The Netherlands. E-mail: m.feiters@science.ru.nl; Fax: +31-243652929; Tel: +31-24-3652016

${ }^{b}$ DUBBLE CRG/ESRF, Netherlands Organization for Scientific Research (NWO), c/o ESRF BP 220, F38043 Grenoble Cedex, France

$\dagger$ Present address: Diamond Light Source Ltd, Didcot OX11 0DE, Oxon, England, UK.
} 